\title{
GEOSPATIAL INFORMATION BASED EMERGENCY RESPONSE SYSTEM FOR GEOLOGICAL DISASTERS
}

\author{
Quanhong Zheng \\ Sichuan Geomatics Center, Ministry of Natural Resources of the People’s Republic of China \\ Technology Innovation Centre for Emergency Surveying and Mapping, Chengdu, Sichuan, China - qhong21@qq.com
}

\author{
Commission III, ICWG III/IVa
}

KEY WORDS: Disaster Management, Emergency Response, Geospatial Information, Geological Disasters

\begin{abstract}
:
Emergency response is the critical phase in disaster management, which can save lives and reduce losses by useful, accurate, and timely information. The surveying and mapping authorities at all levels are making great efforts to meet the increasing demand for geospatial data in disaster and risk management. In this paper, it takes the Sichuan province of China as an example. It presents a geospatial information based emergency response system for geological disasters from the perspective of the provincial mapping authority. The scheme of the response system aims to solve many problems that arose in practical disaster management and to meet the requirements of public safety and government management by geospatial information and services. Built on historical experience and improved through practical application, the system has now effectively improved the workflow in all phases of emergency response. The paper discusses the critical contents of the system's general framework in terms of emergency mechanism, GIS technology, geospatial data, emergency equipment, and professional teams. A case study is applied in this paper to explain how the system makes its function. The end of the article gives an analysis of its benefits and disadvantages and a comparison with other similar systems.
\end{abstract}

\section{INTRODUCTION}

Geological disasters, including earthquakes, landslides, debris flow, etc. have caused severe threats to people's life and property. How to effectively deal with geological hazards is a serious challenge facing all humanity. Disaster management is generally understood in four phases: mitigation, preparedness, response, and recovery (Zlatanova et, 2008). The response phase is the phase occurring after the event to offer useful, accurate, and timely information to reduce or avoid the potential losses from risk and disaster. As the increasing demand for geospatial data in disaster and risk management, surveying and mapping authorities at all levels are making great efforts to improve emergency response capabilities to facilitate better use of geographic information to support decision making.

Sichuan province, with an area of 486,000 square kilometers and a population of 83.4 million, is the fifth largest province in China. It locates in the transitional zone between the QinghaiTibet plateau and the Yangtze River plain. Its topography varies from west to east. The west region is plateau and mountain, where the altitude is more than 4,000 meters. The eastern part is basin and hills, where the elevation is between 1000 and 3000 meters.

Due to its complicated topography, Sichuan is deeply affected by Geohazards. In 2008, a mega earthquake took place in Wenchuan county, causing 68,712 people dead and 17,921 people missing. The quake triggered tens of thousands of coseismic geological disasters on a large scale. In the years after the earthquake, heavy rain repeatedly triggered a large number of landslides, mountain collapses, and debris flows.

In the Wenchuan earthquake relief, the rescue was challenging due to the interruption of communication and transportation. The very first map of the disaster area was crucial for comprehending the disaster situation. The aerial image a few days after the Wenchuan earthquake indicated that the only road to the earthquake-stricken area was blocked severely, and it was difficult to repair within a short time. Meantime the accurate location information is urgent for the airdrop.

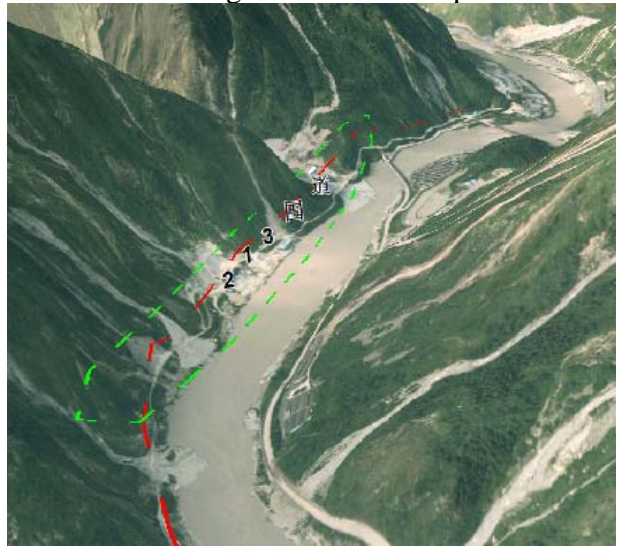

Figure 1. The Aerial Image After The Wenchun Earthquake

On the one hand, was the urgent need for geospatial information and services, on the other hand, were the challenges in data acquisition, analysis, and services. Based on the reflection of fighting the mega earthquake, the provincial mapping agency realized that it was necessary to establish an emergency response system to improve the ability of geospatial information services.

\section{THE GENERAL FRAMEWORK OF THE SYSTEM}

The geospatial information based emergency response system started construction in 2008 based on the experience and lessons learned from the Wenchuan earthquake relief. The emergency response process is a systematic project. The contents of the 
system mainly include five parts, which are emergency response mechanism, historical and current geodatabase, GIS technology, equipment for data acquisition, analysis and services, and talent teams to fulfill the work. These five elements have jointly contributed to supporting disaster management.

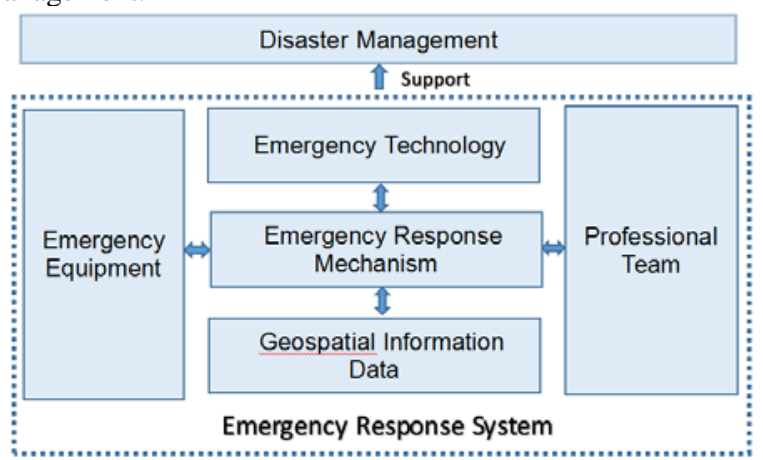

Figure 2. General Framework of the System

\subsection{Mechanism}

Preventing and coping strategies prepared in advance can avoid further expansion or aggravation of the disaster situation and minimize losses. The mechanism is an emergency response plan with a variety of response programs and response levels for different geological disasters. It is a guarantee to prompt and efficient response work.

The emergency plan has covered the contents of emergency organization, response procedures, coordinated response with national, provincial and municipal authorities, daily emergency drills, the requirements for GIS technology and data reserve, equipment management and maintenance, professional team establishment and training, etc.

In case of an emergency, the plan will be initiated immediately with a response level in aligning with the government based on the disaster severity. The program has specified the tasks at each response level to each technical team. Each team will follow the workflow to complete the tasks within the demanded time.

The major GIS tasks in the response phase include images acquisition of the disaster area, data processing, and analysis, thematic map making before and after the disaster, data distribution, on-line GIS services, disaster assessment, etc. These have illustrated in figure 3.

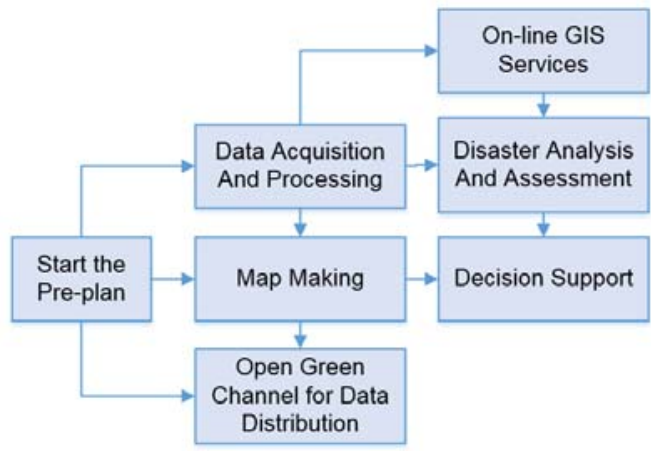

Figure 3. Emergency Response Procedure

The emergency command needs coordination not only with mapping agencies at the national, provincial, and municipal levels but also at all levels of the emergency management department. The emergency plan has defined a coordinated response mechanism for sharing geospatial information with related authorities.

\subsection{Technology}

Scientific technology is a primary driver for improving the capabilities of emergency response with geospatial information and services. Key technologies that involved in the significant process of emergency response include:

(1) Data acquisition under complex terrain and extreme weather.

(2) Emergency command and dispatch.

(3) Geohazards monitoring and warning.

(4) Disaster interpretation and assessment.

(5) On-line public service.

\subsubsection{Data acquisition}

Obtaining data from the affected area is an essential means to understand the disaster situation. Satellite images and aerial photogrammetry images have been valuable resources. The Unmanned Air Vehicle (UAV) system has become an essential technical measure to get the first high-resolution map of disaster information. Due to the complicated terrain and bad weather in Sichuan province, a single drone with a single load operation method is quite limited under the constraints of short-term airspace.

To face this challenge, the Sichuan mapping agency developed the UAV Cluster Disaster Information Acquisition System. The core of the system is heterogeneous multi-UAV collaboration and single-machine multi-load technology. Multiple earth observation hardware and software are all integrated with various UAV Platforms. Based on this technology, three significant advances have been made, including:

(1) Realizing the all-weather rapid data acquisition and on-site processing under the limitation of short-term airspace.

(2) Achieving stable mapping and real-time transmission under complex conditions, especially in extreme cold and high altitude areas.

(3) Developing a spatial analysis and a dynamic simulation system for disasters.

The UAV system is combining with different kinds of UAV, including a plateau UAV, and also a mobile spatial data acquisition system, several aerial cameras, and electro-optical pods. With the integrated function, the operating altitude of the drone can be promoted from 4000 meters to 6500 meters and provides images of exceeding $0.2 \mathrm{~m}$ resolution.

\subsubsection{Emergency Command and Dispatch}

Sichuan provincial mapping agency developed a threedimensional Eergency Command Platform which is a geoinformation system for emergency command. By overlaying on-site image data obtained by UAV, air plane or satellite with three-dimensional terrain, it is possible to to identify the disaster location, study the surrounding terrain, make rescue routes and analyze the disaster trend which is essential for scientific command.

Multi-source, time-series, and high-resolution digital orthophotos, digital elevation models, digital topographic maps, and some thematic data such as geological hazards, earthquake, meteorology, forest fire, etc. are integrated with the 3D Emergency Command Platform. With functions of virtual reality, spatial positioning, and spatial analysis, it is possible to 
conduct disaster analysis, decision deployment, disaster assessment, post-disaster reconstruction planning, etc. Figure 4 is an excavation analysis example of the platform, which calculates the volume of the Tangjiashan dam body and dam lake caused by the Wenchuan earthquake.

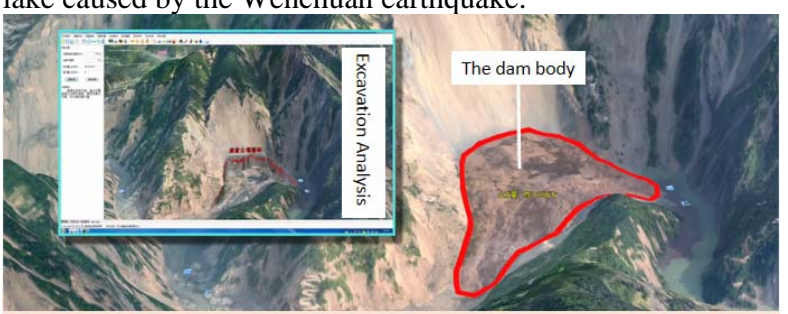

Through excavation analysis, the earth and rock volume of the Tangjiashan dam body is estimated to be about 21 million cubic meters, and the capacity of the dam lake reservoir is estimated to be about 140 million cubic meters.

Figure 4. Excavation Analysis (Technology Innovation Center of Emergency Surveying and Mapping, 2008)

To effectively implement the emergency pre-plan and improve the ability of command and dispatch, an internet-based system is developed by the mapping authority following the task allocation and procedures regulated in the plan. The system provides functions of personnel and equipment management, task management, location monitoring, and emergency consultation. The commander can assign tasks to each professional team, monitor the personnel and vehicle position, and understand the on-going process within the system. Meeting with the front line team is possible by instant message, on-site live video, and conference system.

\subsubsection{Geohazards Monitoring and Warning}

Most potential geohazards locate in high mountains covered by vegetation where it is difficult to identify in advance by traditional manual investigation methods, and these large quantities of risks distribute widely, which are hard to be monitored. Technologies of InSAR, lidar, satellite, and UAV remote sensing are applied broadly to early identification and dynamic monitoring of geological hazards.

Optical remote sensing combining with spaceborne SAR technology can be used for a large-scale general inspection of geological hazards. High-resolution remote sensing images are useful for discovering surface deformation. Time series spaceborne SAR data can help to detect terrain that is deforming slowly.

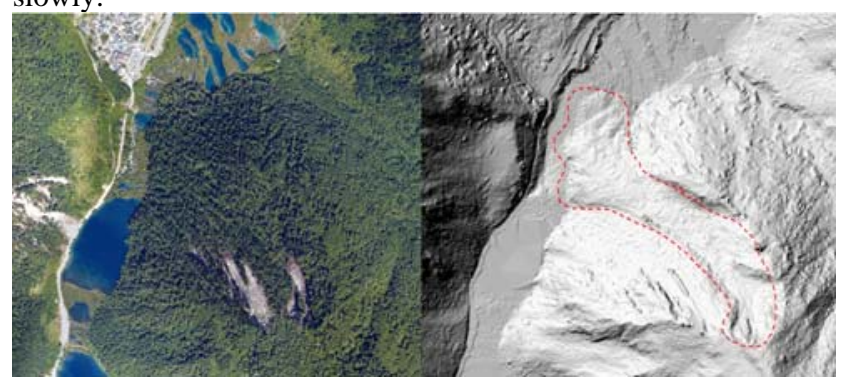

Figure5. Airborne Lidar Data for Geohazards Identification

(Technology Innovation Center of Emergency Surveying and Mapping, 2019)

For a detailed investigation of one specific region, airborne LIDAR and UAV technology are useful. Airborne LIDAR data can help to determine historical "damage" through highresolution DEM, which is obtained from DSM by removing vegetation. UAV aerial photography is excellent for detecting cracks and potential risks.

For significant identified geological hazards, InSAR technology combining with ground sensors can be employed for real-time monitoring and early warning.

\subsubsection{Disaster Interpretation and Assessment}

Artificial Intelligence and deep learning technology have applied in the quick interpretation of disaster information in recent years. The images used for AI learning are UAV images of $0.2 \mathrm{~m}$ resolution covering different types of disasters, including debris flow, earthquake, mountain collapse, etc. First, mark the boundaries of geological hazards, roads, houses, rivers, farmlands, etc., and then design training data set, test data set, and verification data set by a certain ratio such as $60 \%, 30 \%$, and $10 \%$. Randomly sample the training set data to obtain 512 * 512 training samples. Through repeated learning from a large number of these training samples based on the deep learning models, boundaries of different types of disasters, roads, houses, rivers, farmlands, and other geospatial data can automatically be extracted. The extraction accuracy could reach $75 \%$ by test.

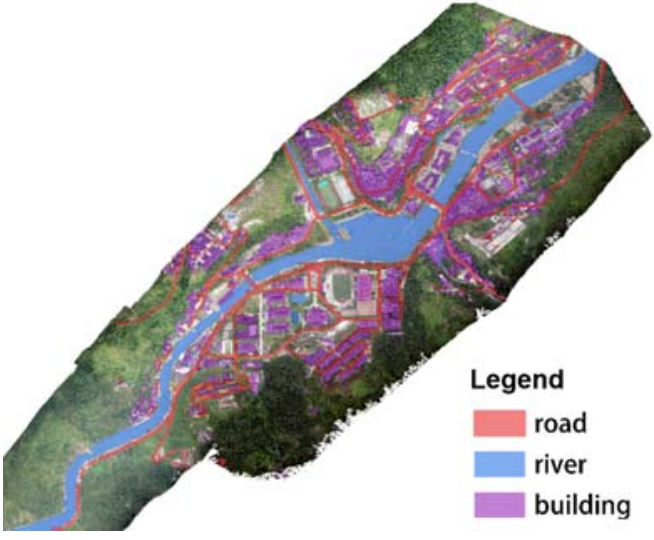

Figure 6. Interpretation Result By AI Technology (Technology Innovation Center of Emergency Surveying and Mapping, 2019) By comparing data before and after the disaster, it is possible to get the scope of the disaster, the number of affected houses, the length of blocked roads and the area of damaged farmland, etc. Statistical graphs and thematic maps could be made based on the comparative analysis, and further to form a disaster assessment report.

\subsubsection{On-Line Public Service}

The geographic information public service platform integrates cross-regional multi-scale geographic information data, providing the public with a variety of online services such as electronic map service, data inventory service, GIS query, and analysis service, etc. Geographic information resources are interconnected through the platform, meeting the needs for geoinformation data from disaster prevention and reduction or emergency handling applications. The platform enhances data sharing between mapping agencies, professional departments, enterprises, and social groups. In case of an emergency event, the platform will release the disaster information, including onsite images at the very first time. 


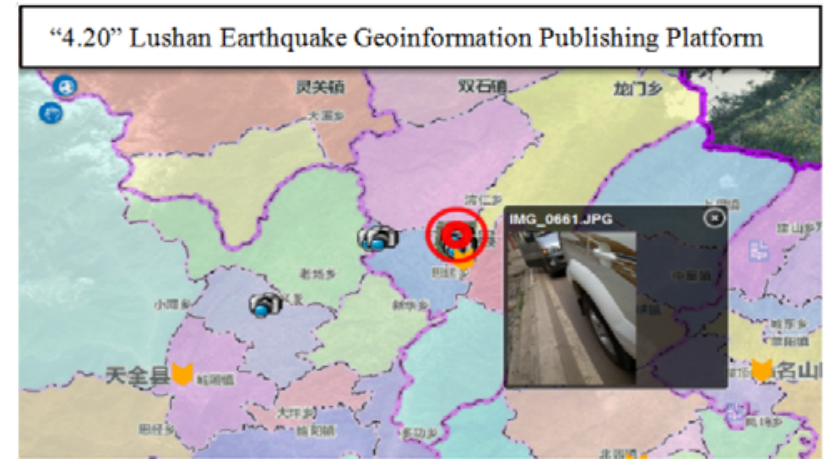

Figure 7. Natural Disaster Publishing Platform (Technology Innovation Center of Emergency Surveying and Mapping, 2013)

\subsection{DATA}

High-resolution Geodatabase is essential for disaster prevention, emergency response, and post-disaster construction. The geodatabase can be classified into three kinds of databases based on the form and contents of the data, which are the fundamental database, thematic database, and on-site sensor database.

The fundamental geospatial database consists of multi-scale, multi-resolution, and time series geographic data. It is the spatial positioning framework for other statistics and thematic data. According to the data types, it includes raw image data, Digital Line Graphic (DLG) data, Digital Orthophoto Map (DOM), Digital Elevation Model (DEM), Digital Grid Map (DRG), and their respective metadata. DLG data includes geodetic data, water systems, residential areas, transportation, pipelines, boundaries, landform, vegetation and place names, etc. Data scales range from 1: 1000,000, 1: 250,000, 1: 50,000, 1: 10,000, 1: 2000 to 1: 500, etc. DOM data includes remote sensing images and aerial photos with spatial resolutions ranging from $30 \mathrm{~m}, 5 \mathrm{~m}, 2.5 \mathrm{~m}, 0.5$ meters, to $0.2 \mathrm{~m}$, etc. DEM data describes the spatial distribution of topography. It is the basis of 3D terrain. The elevation interval ranges from $50 \mathrm{~m}$, $25 \mathrm{~m}, 5 \mathrm{~m}$, and $1 \mathrm{~m}$, etc.

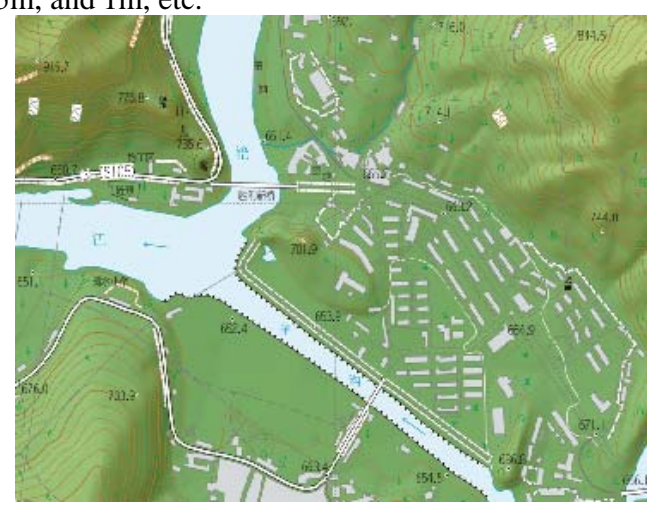

Figure 8. Map Product With DOM, DEM and DLG

Thematic data includes economic statistics data, demographic data, land use data, seismic geology data, meteorological data, hydrological data, forest data, soil data, mine data, ecological, environmental data, and public health data, etc. Key protecting targets such as government departments, schools, key projects, airports, reservoirs, power facilities, energy reserves, and emergency shelters are also critical thematic data.

On-site monitoring data includes UAV image data, 3D laser scanning data, panorama photos, live pictures, audio, video, and so on. On-site data and thematic data combining with geospatial data can help scientific command and deployment.

\subsection{EQUIPMENT}

The equipment for data acquisition, data processing, data storage, and management is essential to fast and provide effective geospatial services.

Data acquisition equipment includes uncrewed helicopter, drone cluster, mobile measuring vehicle, 3D laser scanner, total station, different types of radar and InSAR, etc. The aviation emergency equipment system consists of three kinds of emergency mapping systems. The staffed aerial system is useful for a large area data acquisition, the fixed-wing UAV system useful for crucial area data acquisition, and the unmanned helicopter system is for a small area and flexible purpose.

Equipment for data processing includes rapid processing and mosaic software, digital photogrammetry system, and SAR mapping station, etc. This equipment is for processing of UAV aerial remote sensing image, laser point cloud data, mobile data, tilt aerial photographs, and so on.

Equipment for data storage, management, and output includes Exadata database machine, disk array, database management system, printing and scanning integrated machine, direct plate making machine, different types of printer, and so on.

\subsection{Professional Teams}

Professional technical personnel is the guarantee to fulfill all the tasks. Sichuan mapping agency cooperating with the local university and mountain hazard research institute established the Technology Innovation Center of Emergency Surveying and Mapping to improve the ability of technology research and development. The innovation center brings together a large number of top talents in the industry and focuses on the study of emergency mapping, geological hazards monitoring and warning, and emergency information services.

Besides, the mapping agency also established six professional teams according to different tasks of emergency response, including navigation and location-based services, data acquisition, aerospace image processing, database management, cartography, and hazard monitoring. These technical teams are increasingly improving their capabilities by either academic or practical training.

\section{CASE STUDY}

By perfecting emergency mechanism, improving emergency command and dispatch capability, upgrading emergency equipment, enrich geospatial information data, and strengthen talent team building, the emergency response system on geospatial information and services has been increasingly improved and made great functions in significant geological disasters response over the years, such as Changning 6.0 magnitude earthquake in June 2019, two landslides in Baige village, Jinsha River in October and November 2018 which has formed a barrier lake, the Jiuzhaigou 7.0 magnitude earthquake in August 2017, and Maoxian high mountain collapse in June 2017, etc.

This paper uses the collapse of the high mountain in Xinmo village of Maoxian county as a typical example to explain how this system works in terms of mechanism, technology, 
equipment, data, and professional teams.

At 5:45 AM on June 24, 2017, a high mountain collapse took place in Xinmo village, about $200 \mathrm{~km}$ far away from Chengdu city, the capital of Sichuan province, causing more than 40 households and more than 100 people buried. The Songjianggou River was blocked for $2 \mathrm{~km}$.

The provincial mapping agency initiated the first-level emergency response at 9:30 am. According to the emergency pre-plan, the priority tasks in the response phase are to get the first image of the disaster area, prepare the pre-disaster map, and open a green channel to distribute data for emergency command departments. Following the procedure, a UAV squad headed out to the core disaster area at 10:00 am to obtain the image of the mountain collapse. Meanwhile, the professional map-making team started to prepare the map based on the geodatabase. The 3D Emergency Command Platform was deployed in the provincial government emergency command center to provide geospatial information support for decisionmakers.

The pre-disaster satellite image map and thematic map were accomplished and sent to the national authorities and provincial government at 11:20 am.

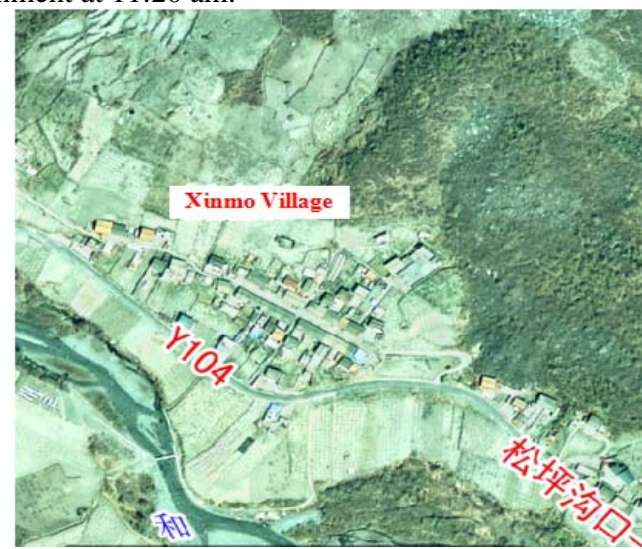

Figure 9. Pre-Disaster Image of Xinmo Village ((Technology Innovation Center of Emergency Surveying and Mapping, 2017)

The post-disaster $0.2 \mathrm{~m}$ resolution image was obtained through the UAV Cluster Disaster Information Acquisition System at 5:35 pm about 8 hours since the start-up of the emergency response. After processing, the first batch of images was transmitted to the provincial emergency command center and loaded to the 3D Emergency Command Platform. Experts from the Technology Innovation Center of Emergency Surveying and Mapping studied the reason for the mountain collapse. They evaluated the damage by comparing before and after disaster images through the 3D geoinformation platform. From the postdisaster image, it is clear that the mountain collapse has caused a barrier lake. A crack with a width of 4-10 meters and a length of 900 meters was detected, which formed a potential instability body. Potential threats to the residential area near the mountain were analyzed. A report on disaster assessment and recommendations for responding to the second disaster was prepared. The research helped the government to make a prompt reaction for resettlement and avoid further damage.

In this mountain collapse case, the whole response process from data acquisition, map making, disaster analysis and assessment to making a responding recommendation report was within 8 hours. The emergency mechanism and plan ensure that the emergency response is carried out in an orderly manner. The
GIS technology and equipment are the means and tools for data acquisition, analysis and service. The geographic data of disaster area aids decision support, and the professional technical teams ensure the effectiveness and timeliness of the tasks.

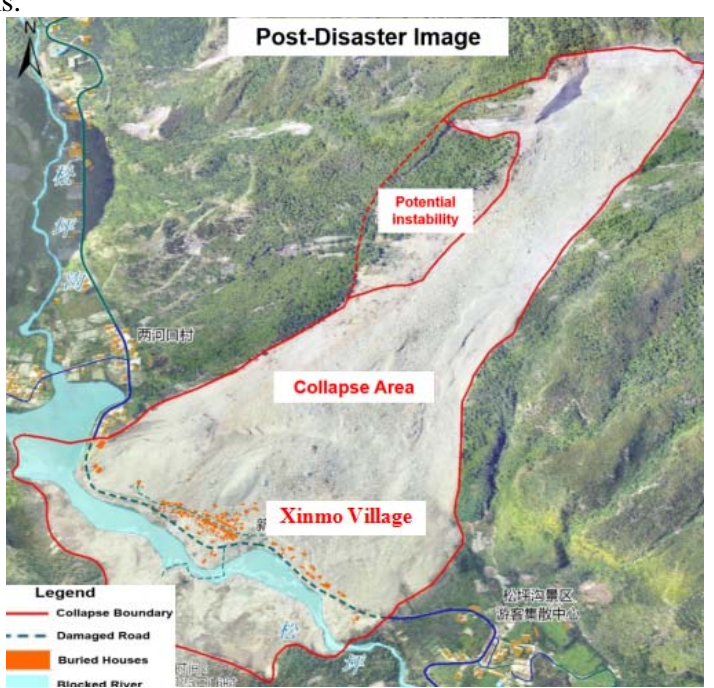

Figure 10. Post-Disaster Image of Xinmo Village

(Technology Innovation Center of Emergency Surveying and Mapping, 2017)

\section{CONCLUSION}

An emergency response is a systematic project. To fully enhance the effectiveness and timeliness in emergency management, it is necessary to consider the joint influence of response mechanisms, GIS technology, geospatial data, emergency equipment, and professional teams. This paper presents the geospatial information based emergency response system in terms of the elements mentioned above, which is applying by Sichuan provincial mapping agency. It aims to share experience in disaster management from the GIS side.

Different countries have different disaster management models. It is hard to say which one is better, depending on their respective policy. China's emergency system is mainly led by the administration, which has established a complete, unified, and grading responsible working mechanism. Administration at a different level has its emergency response plan that is in alignment with the national strategy. The benefits of this administration model are the effectiveness of prompt response and precise command. The weakness is that it is focusing on the emergency rescue whereas the awareness of risk management is insufficient and needs strengthening.

The Pacific Disaster Center of the United States is to make the information more available and time-sensitive after a disaster and assist decision-makers in making timely and accurate decisions. Its mechanism of disaster and risk early warning is becoming more mature. Different hazards can be automatically integrated through the monitoring software to identify risk signals and early warning information.

The Geospatial Information Authority of Japan plays a vital role in disaster prevention and relief. When the disaster occurs, the Japanese government will declare a state of emergency. The Mapping Authority will respond quickly within 10 minutes. The staff will arrive at the emergency headquarters within 30 minutes. The existing map is to be prepared within 1 hour, and 
the post-disaster image is to be provided after the aerial photography aircraft land 4 to 6 hours. The disaster extent and results of damage in the affected areas will be quickly send to the central and local governments.

The New Zealand Geospatial Information Office is to establish a geographic information surveying and mapping infrastructure and to enhance the ability to collect, analyze, and process geographic information. The establishment of the office helps to provide information to more users, so that people are more prepared in the face of major disasters, and improve disaster response capabilities.

Although the geospatial information based emergency response system this paper presented has played a significant role in major geological disaster response over the years, it is more concentrated in response phase. From the prevention perspective, the system still has much room for improvement in the areas of geological hazard identification, monitoring and early warning in the future.

\section{REFERENCES}

Cheng Duoxiang, 2015: Fast Acquisition and Processing of UAV Aerial photogrammetry Data. Surveying \& Mapping Publishing House, ISBN: 978-7-5030-3790-0.

GE Daqing, DAI Keren, GUO Zhaocheng, LI Zhenhong , 2019: Early Identification of Serious Geological Hazards with Integrated Remote Sensing Technologies: Thoughts and Recommendations. Geomatics and Information Science of Wuhan University, 44(7): 949-956.

Meng Yin, Yang Zheng-yin, Zhuo Song, 2013: General Design and Implementation of Surveying and Mapping Emergency Support Services System[A]. Surveying and Mapping, 36(5).

Miao Shude, 2018: Consturction and Application of Emergency Surveying and Mapping Security Service System, taking Gansu Province as example [A]. Mine Surveying, 46(5).

UNGGIM WG-GISD, 2017: Strategic Framework on Geospatial Information \& Services For Disasters. PDF, http://ggim.un.org/documents/UN-

GGIM_Strategic_Framework_Disasters_final.pdf

Yang Sheng, 2009: On the Significance and Urgency of Establishing Emergency Surveying and Mapping Service System through Wenchuan Earthquake[J]. Surveying and Mapping, 32(2): 51-53.

Zlatanova, Sisi (EDT)/Li, Jonathan (EDT), 2008: Geospatial Information Technology For Emergency Response. ISPRS Book Series, Volume 6.

ZHU Qing, CAO Zhenyu, LIN Hui, XIE Weiting, DING Yulin, 2014: Key Technologies of Emergency Surveying and Mapping Service System. Geomatics And Information Science Of Wuhan University, 39(5): 551-555. 